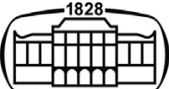

AKADÉMIAI KIADÓ

\title{
Gentile- und Reichsadel am östlichen Rand des Karolingischen Reiches
}

\author{
Béla Miklós Szőke* 『
}

\section{Acta Archaeologica Academiae Scientiarum Hungaricae}

72 (2021) 2, 335-345

\section{DOI:}

10.1556/072.2021.00017

(c) 2021 The Author(s)

\section{ORIGINAL RESEARCH PAPER}

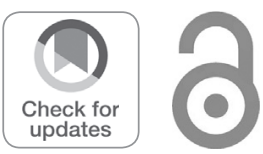

Corressponding author. E-mail: szoke.bela@abtk.hu
Institute of Archaeology, Research Centre for the Humanities, Eötvös Loránd Research Network, Tóth Kálmán utca 4, H-1097 Budapest, Hungary

Received: June 17, 2021 • Accepted: June 22, 2021

\begin{abstract}
At the eastern border of the Carolingian Empire two different groups of elite emerged. When referred to, the individuals in one of the groups were called either by personal names, or by the name of the area they governed; individuals in the other group were called by the name of their people. Members of the first group administered the territorial units of the central area of the former Avar Khaganate just like the Carolingian chief officials and royal vassals in the interior of the Empire. The members of the second group were (indirect) allies of the Avars and had their own tribal prince and gentile nobles. The administrative centres of the Carolingian province Pannoniae developed in synchrony with the inner centres of the Empire, while the centres of power outside the Empire had their own special settlement structures showing a conglomerate of the courts of the tribal nobility.
\end{abstract}

\section{KEYWORDS}

Title of the elite, Carolingian Period, Mosaburg/Zalavár

Die fränkischen Würdenträger an der Spitze der kleineren Regionen des ehemaligen Awarenkhaganats, wie Wonomyr, Liudewit, Borna, Ratimar, Priwina und Chezil $\sim$ Chozil, bezeichneten die Historiker mit großer Vorliebe als dux gentis, die man nach dem ritus gentis ${ }^{1}$ zum Stammesführer/Fürsten wählt. In dieser Frage ist Herwig Wolfram am konsequentesten, nach dessen Meinung die duces an den östlichen Randgebieten ${ }^{2}$ nur gentile Fürsten sein könnten, ${ }^{3}$ die mit Billigung des Kaisers eingesetzt und von Zeit zu Zeit verpflichtet sind, dem Kaiser zu huldigen. In seiner Auslegung besteht ein entscheidender Unterschied zwischen einem gräflichen Mandatsträger und einem mehr oder weniger unabhängigen Stammesführer im religiös-kirchlichen Bereich. Die karolingischen Grafen üben nämlich keine selbstständige Kirchenherrschaft aus und stehen im Rang unter den Bischö-fen. Dagegen leiten die karantanischen oder altmährischen Fürsten von sich die Missionierung ihres Landes ein, die Mährerfürsten versuchen sogar eine eigene „Stammes-kirche“ mit Hilfe Roms und Konstantinopels einzurichten. ${ }^{4}$

Die Administration des Karolingerreiches trifft aber in den westlichen Gebieten des Awarenkhaganats mit großer Wahrscheinlichkeit auf eine Verwaltungsstruktur, die sich

\footnotetext{
${ }^{1}$ Wolfram (1986) 245; Wolfram (1996) 174, wo er 823 von der Einsetzung des Wiltzenfürsten berichtet, die secundum ritus gentis erfolgt sei, als Ludwig der Fromme in einem Streit um Machtfragen zwischen Milegast und Cealadrag entscheidet (Ann. regni Francorum a. 823, RAU I, 132). Nach Štih (2010) 166 ist der Hinweis in den Ann. Fuldenses a. 849 (Rau II, 36) leges et consuetudines Sclavicae gentis gleichbedeutend.

${ }^{2}$ Nach Wolfram (1996) 166 ist für den begrifflichen Inhalt des comes der Fall von Alzeco-Alciosus charakteristisch, der auf den Befehl des Herzogs von Benevent auf seinen Titel dux Vulgarum verzichten muss und den mit dem comes gleichgestellten Rang gastald erhält, nachdem er mit dem gesamten Kriegsvolk seines Fürstentums nach Italien marschiert (Paulus Diaconus, Hist. Langobardorum V 29).

${ }^{3} \mathrm{Zu}$ ihnen zählt er den Soraben- und Abodritenfürsten und die 14 Böhmenfürsten sowie Borna, Liudewit, Rastislav, Zwentibald, Brazlav usw. Wolfram (1996) 169, Anm. 385.

${ }^{4}$ Wolfram (1996) 174-175.
} 
von der gentilen Struktur bereits gelöst hatte. Ihre Anführer, wie der tudun als princeps Pannoniae, ${ }^{5}$ der für das westliche Grenzgebiet verantwortlich ist und in erster Linie die Tributerhebung überwacht ${ }^{6}$ - an dieser Stelle lohnt es sich bemerken, dass bei den Awaren, wie bei anderen Steppenvölkern, nicht nur eine einzige Person den Rang tudun innehaben konnte,$-^{7}$ oder der capcan, princeps Avarorum sind vom Khagan abhängige "Amtsträger“, für die es nach der christlichen Bekehrung kein Problem mehr darstellt, den Erwartungen der karolingischen Administration zu entsprechen, da ihre Aufgabenbereiche eine große Ähnlichkeit mit den Aufgaben des spätantik-fränkischen comes (fränkischen grafio) im Karolingerreich aufweisen, ${ }^{8}$ sofern er nach der bayerischen Lex folgende Aufgaben besitzt: Er hat nach dem Herzog die richterliche Gewalt und ist für seinen comitatus und für das Aufgebot seines Gebietes verantwortlich. ${ }^{9}$ Dagegen wird die gentile Struktur der vom Khaganat zum Teil abhängigen benachbarten slawischen Fürstentümer viel weniger von diesen Einwirkungen berührt und sie werden in unveränderter Form zu Vasallen-Verbündeten des Karolingerreiches. ${ }^{10}$

Die karolingischen Herrscher versuchen sicherlich auch in der neu geschaffenen Provinz Pannoniae - der in den Provin-zen des Reiches von Italien bis zu den Sachsen bereits früher gut bewährten Praxis entsprechend - die Verwaltungs-aufgaben auf die aus den lokalen Anführern ausgewählten Personen zu übertragen und sie an die lokalen Begebenheiten anzupassen, d. h. diese auch auf die im Khaganat über Jahrhunderte hinweg herausgebildeten Nomenklatur abzustimmen. Die aus dem awarischen Khaganat herausgerissenen neuen karolingischen Provinzteilen könnten den früheren territorialen Einheiten

${ }^{5}$ Ann. Laurissenses a. 795 (MGH SS I. 180); Ann. Fuldenses Enhardi a. 795 (MGH SS I. 351). Nach Bóna (1984) 1599 lauten die Bezeichnungen des bereits zum Vasallenfürsten gewordenen Tuduns dux de Pannonia sowie princeps Pannoniarum bzw. princeps Pannoniae.

${ }^{6}$ Bezüglich der Grenzverteidigung lohnt es sich, uns mit jener Angabe zu beschäftigen, wonach „die Chasaren die Völker, die sich ihnen unterwerfen, genauso wie die Westtürken [...] unter die Verwaltung je eines iltbär und je eines tudun gestellt haben“ (Czeglédy, 1967, 86-87).

${ }^{7}$ Pohl (1988) 301.

${ }^{8}$ Wolfram (1995) 214-218, Wolfram (1996) 165-172 hält den comes für den lokalen Vertreter des Königs (Kaisers), den man in Baiern auch grafio nennt: „Ein Graf stand zwar an der Spitze eines militärischen Aufgebots, das sich als Personenverband darstellte. Da aber diese Menschen in einem bestimmten Gebiet wohnten, war die Beauftragung des Grafen regional begrenzt“ (Wolfram, 1995, 216).

${ }^{9}$ Lex Baiwariorum II. 4-6 und II. 14. Dies setzt sich ungebrochen auch in der Karolingerzeit fort, als der Graf ein königliches Mandat bekleidet. Sein Aufgabenbereich entspricht dem des Bischofs als ministerium, was nicht nur die Funktion des Grafen, sondern die seines gesamten Erfüllungsstabes mitsamt den Mitarbeitern, Helfern und dem ihm unterstellten Richter und seinen Gütern bedeutet (Capitulare Bawaricum c. 4).

${ }^{10}$ Wolfram (1986) 249-249 stellt den altmährischen Fürst Zwentibald (Swatopluk) als eines der ausdrucksvollsten Beispiele für den gentilen Fürst dar. entsprechen und die mit ihrer Verwaltung Betrauten nach der Bekehrung und dem Vasalleneid - bleiben die Gleichen.

\section{TITULATUR DES HOCHADELS AM ÖSTLICHEN RANDGEBIET DES KAROLINGISCHEN REICHES}

Die Unterschiede in dem Rang und der Funktion der führenden Persönlichkeiten der östlichen Randgebiete des Karolingischen Reiches bemerken auch die zeitgenössischen Schriftkundigen. Sie differenzieren nämlich die Anführer der Region deutlich in Hinblick auf den Namens- und Titulusgebrauch. Bei den Mitgliedern der einen Gruppe erscheint nach der Rangbezeichnung $d u x$, princeps, rex stets auch der Name des angeführten Volkes, wie z. B. im Fall der donaubulga-rischen, altmährischen, ${ }^{11}$ böhmischen und abodritischen Fürsten. In der anderen Gruppe fehlt dies vollkommen und man erwähnt sie nur mit dem bloßen Eigennamen - das ist mit der Praxis der im Reichsinneren einen Verwaltungsposten bekleidenden anderen karolingischen Hauptamtsträger und königlichen Vasallen völlig synchron. In diesem Bereich bildet sich erst im Verlauf des 9. Jahrhunderts stufenweise ein so konstantes Attributssystem heraus, in dem der Eigenname bereits mit der Funktion und/oder dem Gebiet seiner Ausübung fest verankert ist. ${ }^{12}$ Wenn man - in sehr seltenen Fällen - dem Eigennamen einen der Würdennamen dux oder rector doch hinzufügt, dann geschah es nie mit dem Volksnamen, sondern man assoziiert diesen mit den von ihnen verwalteten Gebieten und/oder deren Zentren, wie im Fall von Liudewit (Pannonia inferioris, Siscia), Priwina (urbs paludarum) und Borna (Dalmatia et Liburnia).

Es gibt aber auch zwischen den Mitgliedern beider Gruppen einen markanten Unterschied in ihrer Verhaltensweise dem Reich gegenüber bzw. darin, in welcher schriftlichen Quellengruppe sie eher erwähnt werden. Den foederati-Fürsten der ersten Gruppe, wenn sie persönlich oder mittels ihrer Gesandtschaften an den Reichsversammlungen teilnehmen, gedenken auch die Annalen als diplomatisches Ereignis. Die Mitglieder der zweiten

\footnotetext{
${ }^{11}$ Conversio c. 10 (Wolfram, 1979, 50; Lošek, 1997, 120): a Moimaro duce Maravorum supra Danubium. - Den Unterschied, wonach Liudewit und Priwina gegenüber dem mährischen Herrscher keinen Sondernamen aufweisen, bemerkt auch Wolfram (1986) 250. Er erklärt es aber damit, dass dies eine militärische Formation sei, „die sich durch eine hohe Mobilität auszeichnet und die sich um Gefolgschaften, praetoriani, bilden“.

${ }^{12}$ Wolfram (1995) 216; aber sogar bei den Mächtigsten, wie bei dem Traungaugraf Graman und Wilhelm I., bleiben die darauf hinweisenden schriftlichen Beweise aus.
} 
Gruppe erwähnt man aber nur, wenn sie bei diesen Versammlungen mit speziellen Aufgaben betraut werden. Ansonsten begegnen wir ihnen nur in den Privatbriefen und Schenkungsurkunden. ${ }^{13}$

Der Unterschied in der Namensverwendung grenzt sich auch geographisch gut ab. Das Herrschaftsgebiet der mit dem Volksnamen zusammen erwähnten Fürsten der ersten Namensgruppe erstreckt sich außerhalb des einstigen Awarenkhaganats, während der Bereich der zweiten Gruppe der Anführer sich innerhalb dessen befindet. $d u x$ der Name seiner Provinz, Forum Iulii. Die Funktion eines karolingischen Amtsträgers von Liudewit betont auch der Wortgebrauch Thegans in Vita Ludwig des Frommen, als er über ihn als von einem vom karolingischen Herrscher betrauten rector inferioris Pannoniae spricht. Auch den Liudewit folgenden Ratimar erwähnen die zeitgenössischen Quellen mit seinem bloßen Eigennamen oder mitsamt dem Titulus $d u x$. Dem Titel $d u x$ fügt man jedoch nicht einmal den Provinznamen, geschweige denn den Volksnamen hinzu. Es wird nur durch den Textzusammenhang deutlich,

cum Wonomyro Sclavo in Pannonias hringum gentis Avarorum (Ann. regni Francorum a. 796, RAU I. 64)

cum Wonomiro Sclavo (Ann. Tiliani a. 796, MMFH I. 58; Reginonis Chronicon a. 796, MMFH I. 133; Annalista Saxo a. 796, MMFH I. 246)

cum Wanomiro Sclavo (Ann. Mettenses piores a. 796, MMFH I. 54)

contra Liudewitum quoque Sclavum in Pannonia rebellantem (Ann. Fuldenses a. 819, MMFH I. 87)

contra Liudwidum Sclavum rebellantem (Herimanni Augiensis Chronicon a. 819, MMFH I. 162)

propter Liudewiti (Ann. regni Francorum a. 819, RAU I. 118)

de Liudewiti (Ann. regni Francorum a. 820, RAU I. 120)

de bello Liudewitico (Ann. regni Francorum a. 821, RAU I. 124)

Liudewitus Siscia civitate relicta (Ann. regni Francorum a. 822, RAU I. 128)

contra Liudewitum in Pannoniam (Ann. Fuldenses a. 820, MMFH I. 88)

Liuteviti rectoris inferioris Pannoniae (Anonymi vita Hludowici c. 31, RAU I. 306);

contra Liudeviti (Anonymi vita Hludowici c. 32, RAU I. 308)

de infestatione Liudeviti, ... ad Luidevitem (Anonymi vita Hludowici c. 33, RAU I. 310)

Liudeviti tyranni (Anonymi vita Hludowici c. 36, RAU I. 316);

Liudewiti, ducis Pannoniae inferioris (Ann. regni Francorum a. 818, RAU I. 116)

Sequenti anno (820) exercitum suum misit adversus orientales Sclavos, quorum dux nominabatur Liduit (Thegani Vita Hludowici imperatoris c. 27, MMFH II. 25)

Ericus dux foroiulensis (Ann. Tiliani a. 796, MMFH I. 58)

Heinricus dux Foroiulanorum (Reginonis Chronicon a. 796, MMFH I. 133);

Bornae, ducis Guduscanorum (Ann. regni Francorum a. 818, RAU I. 116);

Borna, vero dux Dalmatiae (Ann. regni Francorum a. 819, RAU I. 118);

Borna, Bornae (ohne Titulatur) (Ann. regni Francorum a. 819, RAU I. 120; Anonymi vita Hludowici c. 32-33, RAU I. 310)

ad Liudemuhslum avuncumum Bornae ducis (Ann. regni Francorum a. 823, RAU I. 132)

Ratimari ducis; Ratimarum ducem (Conversio c. 10, Wolfram 1979, 52)

contra Ratymarum (Ann. Iuvavenses maximi a. 838, MMFH IV. 383)

contra Ratimarum (Ann. sancti Rudberti Salisburgensis a. 838, MMFH IV. 402; Auctarium Garstense a. 838, MMFH IV. 425)

Ratomaro; Rathomarus regulus (Ioannis Aventini Ann. Boiorum IV. XII 7, 13, MMFH I. 343)

Den Anführer von Pannonia inferior, Liudewit, bezeichnet man entweder mit dem Attribut sclavus, wie vor ihm bereits Wonomyr, oder man erwähnt ihn noch öfter ohne jedweden Titel und Rang, nur mit seinem Eigennamen. Wenn an den Namen Liudewits - ausnahmsweise - doch der Rang dux angeschlossen wird, so wird nicht der Stammesname, sondern nur der Name der ihm anvertrauten Provinz Pannonia inferior angehängt. Das Gleiche erfolgte auch im Fall seines Lehnsherrn, des Herzogs Erich von Friaul: Bei ihm steht ebenfalls hinter dem Würdennamen

\footnotetext{
${ }^{13}$ Deshalb ist es nicht verwunderlich, dass die den awarischen Khagan vertretenden Gesandten von denen der an den Reichsversammlungen Teilnehmenden ab dem Zeitpunkt (828) verschwanden, als man auch die letzten gentilen Formationen in den östlichen Provinzen des Karolingerreiches, also in Karantanien und in Pannonien, liquidiert.
}

dass er die verantwortliche Person für das einst Liudewit anvertraute Pannonia inferior ist. Bei der Titelverwendung ist Borna außerhalb des ehemaligen Khaganats ein Grenzfall, der bei seiner ersten Erwähnung noch Fürst der Guduscaner ist. Später erwähnt man ihn nur ohne den Namen seines Volkes, mit dem bloßen Eigennamen oder als Fürst von Dalmatien und Liburnien.

Mit dem Titulusgebrauch Priwinas und seines Sohnes Chezil Chozil befasste sich bereits Ágnes Cs. Sós ausführlich. ${ }^{14}$ Auch ihr fällt auf, dass man Priwina nicht nur in der Conversio, sondern auch in den meisten Urkunden ohne Titulus, mit bloßem Namen erwähnt, obwohl ihm von Anfang an der Würdenname dux gebührend gewesen wäre,

\footnotetext{
${ }^{14}$ Cs. Sós (1973) 40, Tab. 1.
} 
wenn die Aussage richtig wäre, dass Priwina als gentiler Fürst $(d u x)$ von Nitra zu Ratpod flüchtet und als solcher ein Lehnsgut in der Umgebung des Flusses Zala erhält. ${ }^{15}$

Über die Verbindung Priwinas zu seinem „Volk“ ist der Wortgebrauch des Verfassers der Conversio am verräterischsten: Dieses Volk besitzt nicht nur keinen Namen, sondern auch bei ihm erfolgt nicht einmal ein Hinweis auf gens oder natio, den der Verfasser mit Recht und korrekt verwenden würde, wenn Priwina durch Blutsbande mit seinem Volk verbunden wäre ${ }^{16}$ - auch wenn diese nur durch den von Wolfram angenommenen colluvies gentium zustande kommen. Demgegenüber benutzt er die Bezeichnung populus, als er darüber berichtet, dass Priwina bei seiner Ansiedlung et circumquaque populos congregare („sammelte Völker von überall / ringsum Leute um sich"). ${ }^{17}$ Dies geschieht offensichtlich aus dem Grund, weil der Terminus populus den im politischen Gesellschaftsbegriff der antiquitas (civilis societas) wurzelnden Charakter seines Volkes getreuer wiedergibt, als der die Gemeinschaft der „natürlichen“ Abstammung oder Verwandtschaft ausdrückende gens bzw. die engere regionale Gruppe bezeichnende natio. ${ }^{18}$ Priwina versammelt offensichtlich kein „Volk“ um sich - im engsten Sinne des Wortes. Es ist auch nicht die Rede von colluvies gentium, weil er keine Art von Stammesverband etabliert. Wie gerade die in diesem Gebiet freigelegten endawarenzeitlichen Gräberfelder beweisen, findet er hier im Vergleich zur Siedlungs- und Bevölkerungsdichte der Epoche genügend Menschen. Aus einem solchen Blickwinkel ist der Raum sogar überrepräsentiert - was für Priwina für die Auswahl des Ortes natürlich ausschlaggebend sein könnte. Daher müssen wir unter populos congregare' vielmehr die Anwerbung der spezialisierten Dienstleute für den Betrieb des frühfeudalen Zentrums Mosaburg/Zalavár verstehen. ${ }^{19}$

\footnotetext{
${ }^{15}$ Cs. Sós (1973) 41 nimmt eindeutig gegenüber der Auslegung von gentilis dux Stellung, als sie erörtert, „dass Priwinas und Kozels Benennung als $d u x$ und comes nichts anderes bedeuten dürfte, als dass sich ihre Stellung nicht von der der gleichfalls bald als $d u x$, bald als comes bezeichneten fränkischen Grafen unterschied, die dem Präfekten unterstellt waren“. Dagegen handelt es sich nach Wolfram (1986) 251 bei Liudewit und Priwina um zwei slawische Fürsten unter fränkischer Oberhoheit, gegen die wiederum Liudewit ankämpft. „Priwina ordnete sich völlig in das fränkische Lehenswesen ein, um es als Schutz und Legitimierung sowohl gegenüber den fränkischen Mandatsträgern wie gegenüber seinen eigenen Leuten zu gebrauchen. Hingegen war Priwinas Position niemals die eines fränkischen Mandatsträgers im ComesRang.“

${ }^{16}$ Charakteristisch ist bereits die Namensverwendung der Conversio Bagoariorum et Carantanorum selbst, die bei der Schilderung der Mission in Pannonien nicht über ein konkretes Volk berichtet und besonders im Fall von Priwina und Chezil keine ihnen untergeordnete ethnische Gruppe mit eigenem Namen benennt - entgegen der Schilderung der Bekehrung bei den Karantanen und Baiern, wo die Benennung mit einem Volksnamen kein Problem darstellt.

${ }^{17}$ Wolfram (1979) 53; Lošek (1997) 122; Györffy (1958) 154.

${ }^{18}$ Szücs (1997) 42-50.

${ }^{19}$ Populus bedeutet in diesem Zusammenhang sicherlich kein „Kirchenvolk“; vgl. Hellmann (1964); Wolfram (1979) 90.
}

Nur in einem einzigen Fall können wir einen Titulus neben dem Namen Priwinas lesen: Als Ludwig der Deutsche auf die Bitte des Abtes Otgar von Nieder-Altaich hin eine Schenkung Priwinas bei Salapiugin bestätigt, die sein getreuer Fürst (fidelis dux noster) ihnen ,aus seinem Gut innerhalb seines Herzogtums" (de sua proprietate in suo ducato) schenkt. ${ }^{20}$ Indem der Herrscher Priwina in einer von ihm ausgestellten Urkunde als $d u x$ und sein Gebiet als ducatus benennt, drückt er auf höchster offizieller Ebene aus, was er von dem gesellschaftlichen Prestige und Rechtsstatus Priwinas hält. ${ }^{21}$ Dass es sich bei diesem $d u x$ sicherlich nicht um die Entsprechung von dux gentis, sondern um ein Mitglied der Reichsaristokratie handelt, beweist gerade die Schenkungsurkunde, da Priwina auch noch sein Eigenbesitz nur mit Erlaubnis und Einwilligung des Königs dem Kloster von Nieder-Altaich schenken darf. $^{22}$ Hinsichtlich der Inhaltsänderung der Titulatur während des Jahrhunderts ist es lehrreich, dass comes Chezil nach seinem Tod, in einer Urkunde von 876-880, ebenfalls als $d u x$ erwähnt wird. ${ }^{23}$ Wenn Chezil tatsächlich als Erbe des gentilen Fürstentums den Platz seines Vaters eingenommen hätte, dann hätte ihm ansonsten der gleiche $d u x$-Titulus gebührt, wie seinem Vater. Die Bezeichnungen humilissimus comes und comes de Sclavis bedeuten nicht die Erweiterung seiner Rechtsstellung, ${ }^{24}$ auch nicht die Erlangung des Grafmandats neben dem Fürstentum, sondern gerade das Gegenteil ist der Fall:

\footnotetext{
${ }^{20}$ Die Urkunde wird am 20. März 860 in Regensburg ausgestellt und die Grenzen des Besitzes werden ausführlich beschrieben: ad Salabiugiti infra terminos istos: in orientem ultra Salam fluviolum usque in Slougenzinmarcham et Stresmaren et sic sursum per Salam usque ad Uualtungesbah et sic inde usque in Hrabagiskeit et ad Chirihstetin (MMFH III, 54). Bei diesem Akt ist Priwina wahrscheinlich nicht persönlich in Regensburg anwesend, da man nach der narratio der Urkunde dem Abt von Nieder-Altaich in seiner dispositio all das bestätigt, was Priwina bereits früher dem Kloster geschenkt hatte; vgl. Štih (1994) 211, Anm. 9. Die Schenkung bei Salapiugin ist auch deshalb interessant, weil um 852853 der Salzburger Erzbischof „zu Salapiugin eine Kirche zu Ehren des heiligen Rupert weihte. Diese übergab Priwina mit allem Zubehör an Gott, den heiligen Petrus und den heiligen Rupert, damit davon die Männer Gottes aus Salzburg auf ewig den Fruchtgenuss haben sollten“ - Conversio c. 11 (Wolfram, 1979, 54-55; Lošek, 1997, 126-127). Dadurch wird auch diese Schenkung Teil jener Besitzungen, die Ludwig der Deutsche von demjenigen, dem Priwina sie gab, nimmt, wie die Conversio c. 12 berichtet: „ausgenommen jene Besitzungen, die offenkundig zum Bistum der Salzburger Kirche gehören, also dem heiligen Petrus, dem Apostelfürsten und dem heiligen Rupert“ (Wolfram, 1979, 54-55; Lošek, 1997, 128-129).

${ }^{21}$ Angefangen mit Hans Pirchegger (1912) machten bereits viele, so Herwig Wolfram und auch Karl Brunner, darauf aufmerksam, dass die Benennung in der königlichen Diplomatie „einen höchst offiziellen Charakter besitzt“; vgl. Štih (1994) 216.

${ }^{22}$ Štih (1994) 216.

${ }^{23}$ MMFH III, 69, Nr. 38: quod Chezil dux quondam pro remedio animę suae ad predictum sanctum condonavit...

${ }^{24}$ Wolfram (1996) 334.
} 
Es bedeutet endlich die vollberechtigte Aufnahme in die Reichsaristokratie. $^{25}$

\section{RAUMORDNUNG DES HERRENSITZES - CIVITAS MOSABURG}

Der sich in der Titulatur des Hochadels zeigende Unterschied erscheint auch in der Raumordnung der Herrensitze des östlichen Randgebietes des Karolingischen Reiches. Heute ist die Erforschung des Sitzes von Priwina und Chezil in Mosaburg/Zalavár (Abb. 1) so fortgeschritten, dass die Entwicklungsphasen der Makrostruktur dieser Siedlung bereits zuverlässig beschrieben werden können.

Der am Anfang der 840er Jahre errichtete, befestigte Sitz Priwinas lag auf dem südlichen Teil der L-förmigen Burginsel, im sumpfigen Tal des Flusses Zala. Bei der Auswahl der Lage seines Sitzes folgt er wahrscheinlich noch dem Schema der altmährischen Zentren, von wo er vertrieben wurde. Sicherlich geschah es nicht willkürlich, dass der Verfasser der Conversio den Sitz Priwinas - der zeitgenössischen Praxis entsprechend - nicht als curtis, ${ }^{26}$ sondern als munimen und castrum nennt, und auch der Salzburger Erzbischof Adalwin kommt später in castro Chezilonis noviter Mosapurc vocato $\mathrm{zu}$ Besuch. ${ }^{27}$ Der Verfasser der Conversio hat also dadurch, dass er von der üblichen Terminologie der Epoche abweicht und von munimen/castrum berichtet, von einem solchen Bau also, den „Festung, Schanze, Graben“ umgibt, betont auf den Befestigungscharakter des Herrensitzes hingewiesen. Jene Orte, die mit Gewissheit befestigt waren, erwähnte man auch woanders nicht als curtes, sondern als castra. ${ }^{28}$ Außerhalb des Befestigungsgrabens des Herrensitzes wurde zuerst die rechteckige Stabkirche Johannes des Täufers, eine Hallenkirche mit geradem Chorabschluss und ein palastartiges Holzgebäude, wahrscheinlich für den Salzburger Chorbischof Osbald, gebaut. Um die Holzkirche und den Holzpalast des Chorbischofs war die Insel noch undicht besiedelt. Diese erste Phase endet 850, als die Eigenkirche Priwinas

\footnotetext{
${ }^{25}$ Nach Tellenbach (1979) 280 ist der $d u x$ im karolingischen Ostreich kein offizieller Titel, sondern entweder rein technisch mit „militärischer Führer" zu übersetzen ist, oder eine unstabile Bezeichnung darstellt, die selten in den Urkunden besonders wichtigen Leuten beigelegt werden kann, dem König besonders nahe stehenden Helfern am Hof und im Land draußen, vor allem in den Grenzbezirken. Auch ducatus können neben Stammesländern andere Regionen mit einer gewissen Tradition heißen Aller Wahrscheinlichkeit nach konnte Ludwig der Deutsche den Titel $d u x$ und ducatus in seiner Urkunde vom 20. März 860 bereits in diesem Sinn benutzen.

${ }^{26}$ Nach Reutter (Reutter, 1912, 7) bezeichnet curtis ursprünglich ein königliches Wirtschaftszentrum, Gutshof, wo man die Ernte lagerte und wo die königlichen Mandatsträger und Beamten wohnten. Er zählt 70 Siedlungen im Gebiet der Ostmark, die man im 9. Jahrhundert in einer Urkunde oder in anderen Schriftquellen als curtis, civitas, oppidum, locus usw. nannte. Siehe A. Gauert in RGA Bd. 5 (1982) 105-112 (Curtis); Koller (1988) 8-17; Kahl (2003) 368-369.

${ }^{27}$ Conversio c. 13 (Wolfram, 1979, 56; Lošek, 1997, 132).

${ }^{28}$ Reutter (1912) 9
}

innerhalb des Herrensitzes zu Ehren der heiligen Gottesmutter Maria geweiht wurde. Das Siedlungsbild dieser Phase bestimmte also der Befestigungsgraben und südlich davon eine Wallanlage, die die Insel in zwei große Einheiten teilte: In Süden lag der Sitz von Priwina und Chezil und nördlich davon die Kirche Johannes des Täufers mit dem Holzpalast des Chorbischofs.

In der Mitte der 850er Jahre wurde die Wallfahrtskirche des Märtyrers Hadrian östlich von der Kirche Johannes des Täufers, in der geometrischen Mitte der Insel gebaut, gleichzeitig wurde die bisherige Siedlungsstruktur grundsätzlich umgestaltet. Der Befestigungsgraben wurde größtenteils zugeschüttet, des Weiteren südlich von der Hadrianskirche zwei Holzpaläste für den Salzburger Erzbischof und seine Gefolgschaft, nördlich davon die Werkstätten, Speichergruben und Wohnplätze der Handwerker und Dienstleuten errichtet und der ganze Bezirk mit einer Palisadenmauer abgegrenzt. Dadurch entstand die Pfalz des Salzburger Erzbischofs und die Insel teilte sich auf drei Teilen: Das Gebiet nördlich vom Herrensitz Priwinas und Chezils bestand teils aus einer mit Palisadenmauer umgrenzten erzbischöflichen Pfalz und östlich davon aus einem bisher völlig unerforschten, jedoch dicht besiedelten Teil. Am Ende dieser Zeitperiode wirkten Konstantin (Kyrill) und Method in Mosaburg als Lehrer, danach einige Jahre lang Method als Erzbischof Pannoniens mit Sitz in Mosaburg - damit hängt wahrscheinlich ein kleinerer innerer Umbau der Hadrianskirche zusammen. Deshalb kann man wohlbegründet annehmen, dass der Umbau des Innenraumes der Wallfahrtskirche in Mosaburg, die Abtrennung des Hauptschiffes, mit der Tätigkeit jenes Method in Zusammenhang steht, der diesen Kirchenraum bewusst so hätte gestalten können, dass die Kirche nicht nur als Wallfahrts- und Friedhofskirche, sondern auch als Bischofskirche funktionierte. $^{29}$

\section{DIE AUFGABE DER KIRCHEN VON MOSABURG}

Die Aufgabe der Kirchen von Mosaburg war viel komplexer, als die der altmährischen Kirchen. Sicher ist, dass als Erzbischof Liupram die Hadrian-Wallfahrtskirche in der Stadt Priwinas erbauen ließ, errichtete er nicht einfach eine honorabile ecclesia, sondern schaffte er - die immanenten Möglichkeiten der beiden anderen, bereits stehenden Kirchen (die Eigenkirche Priwinas zu Ehren Maria und das Baptisterium des Heiligen Johannes des Täufers) ausnutzend - einen breiteren baulichen und liturgischen Rahmen, in dem die einzelnen Glieder „in der Vollziehung der gottesdienstlichen Handlungen aufeinander angewiesen sind “ ${ }^{30}$ In

\footnotetext{
${ }^{29}$ In diesem Zusammenhang lohnt es sich, den Grundriss der III. Kirche von Mikulčice (sog. Basilika) in Erinnerung zu rufen, bei der innerhalb des einschiffigen Raumes die zwei langen Streifenfundamentierungen vielleicht eine ähnliche Funktion besaßen. Zu den Kirchen von Mikulčice s. Poláček (2009) 421.

${ }^{30}$ Lehmann (1962) 21.
} 

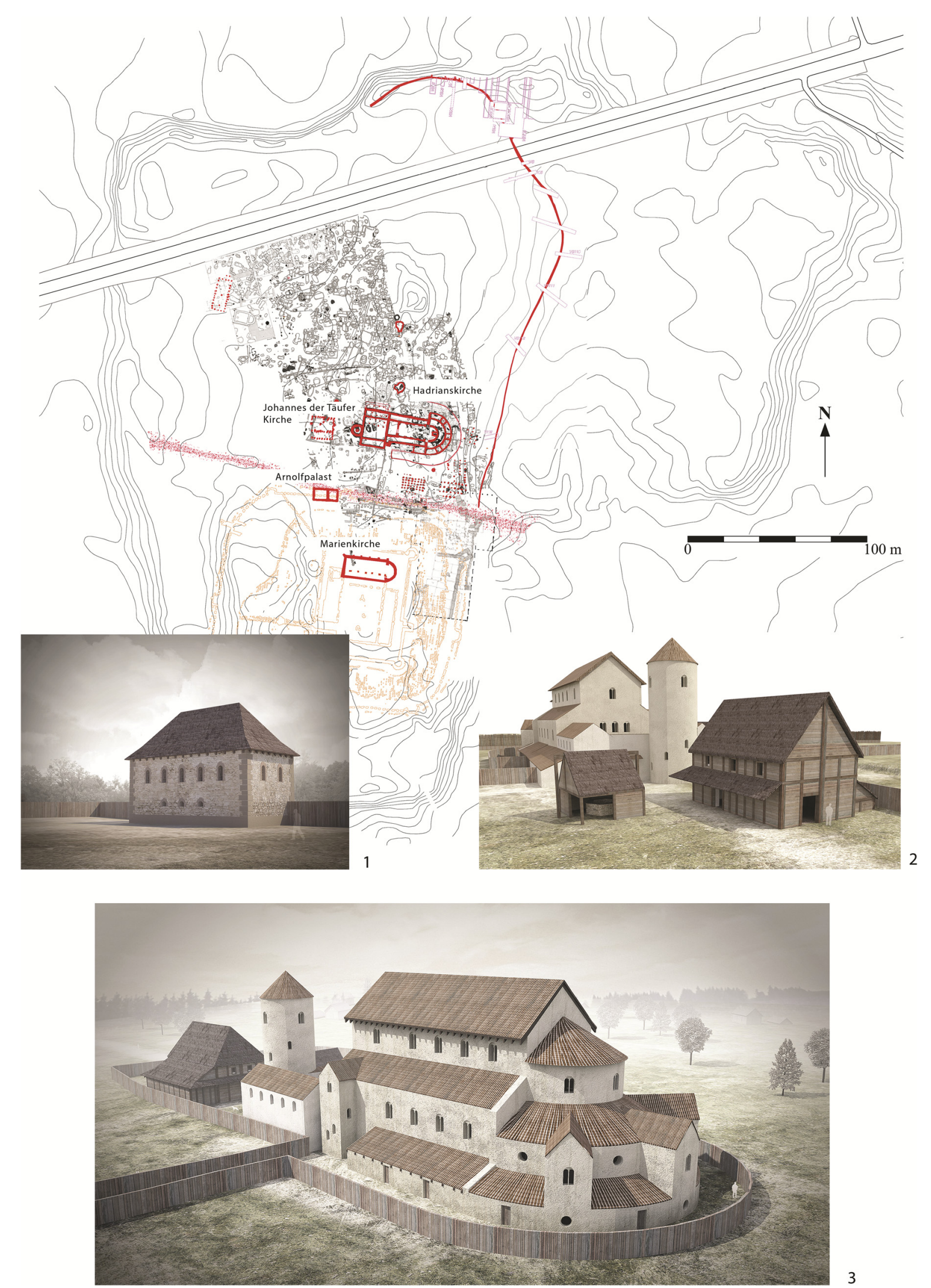

Abb. 1. Zalavár-Vársziget (Burginsel). 1. Arnolf-Palast; 2. Kirche des Johannes des Täufers; 3. Wallfahrtskirche des Märtyrers Hadrian 
dieser Kirchenfamilie erweitern sich die Aufgaben der einzelnen Kirchen. Die Hadrian-Wallfahrtskirche erfüllte die Aufgabe einer Memorialkirche, die aber gelegentlich als Thron- und Erscheinungskirche des Bischofs benutzt wurde, wo der Bischof die rechtliche Seite seines Amtes als Stellvertreter Christi ausübt, die Firmung feiert, Priester weiht und kirchliche Befehle und Rechtsprüche verkündet, während die Marienkirche dem eigentlichen Gemeindegottesdienst zur Verfügung steht. Zwischen diesen zwei Kirchen erscheint das Baptisterium als dritter Sakralraum. Die Mosaburger Dreikirchengruppe ist eine Memorialfamilie, die gelegentlich auch den Kriterien der episkopalen Kirchenfamilie entspricht. Liupram erhebt Mosaburg mit dieser Kirchenfamilie im reinsten Sinne ${ }^{31}$ auch in geistiger Hinsicht auf das Niveau der Reichszentren.

\section{MOSABURG REGIA CIVITAS - KÖNIGSPFALZ DES KÖNIGS ARNOLF}

Am Ende der 880er Jahre kam der König Arnolf beinahe jährlich nach Mosaburg. Er stellte mehrmals Urkunden hier aus (888: urbs Mosaburc, ${ }^{32}$ Mosapurhc, ${ }^{33}$ 889: Mosapurg ${ }^{34}$ ), einmal sogar, in einer Urkunde von 890, bezeichnete er den Ort als Mosaburc regia civitate. ${ }^{35}$ Der Herausbildung der Königspfalz folgte wieder eine neue Siedlungsstruktur: Die Holzpaläste der erzbischöflichen Pfalz wurden abgerissen und an ihrer Stelle für die Bestattungen um die Marien- und Hadrianskirche Platz geschaffen. Die Fundamente des Königspalastes wurden teils auf dem Gebiet des früheren, bereits zugeschütteten Befestigungsgrabens gebaut und eine neue Palisadenmauer um die Königspfalz herum errichtet. In dieser Zeit bebaute man das früher noch frei gelassene Gebiet vor der Hadrianskirche und um die Kirche Johannes des Täufers mit ebenerdigen Häusern und anderen Siedlungsbefunden der Königspfalz.

Nordwestlich von der Westfassade der Marienkirche befinden sich Fundamente eines großen rechteckigen Steingebäudes, die sehr wahrscheinlich zum Königspalast Arnolfs gehörten. Eine Trennwand teilte das Gebäude in einen kleineren und einen größeren Saal, und an der Südseite hat sich ein Vorraum mit gelbem Estrich und mit Palisadenmauern dem Gebäude angeschlossen. Mit einer Palisadenmauer könnte man ebenfalls einen unmittelbar dem Palast zugehörigen, geschlossenen Hof abgetrennt haben.

Das Alter der Palastfundamente wurde durch die Schichtverhältnisse und durch die spezielle Zusammensetzung des Mörtels (stark mit Sand und wenig Kalk und Ziegelschutt vermischt), ferner von den Bruchstücken eines

\footnotetext{
${ }^{31}$ Lehmann (1952/53) 131-144; Lehmann (1962) 21-37.

${ }^{32}$ MGH Dipl. Arnolfi 30, Nr. 19.

${ }^{33} \mathrm{MGH}$ Dipl. Arnolfi 31, Nr. 20.

${ }^{34}$ MGH Dipl. Arnolfi 62, Nr. 43.

${ }^{35}$ MGH Dipl. Arnolfi 112-114, Nr. 75.
}

blauen Glasbechers mit Goldfolienverzierung bestimmt. Es konnte frühestens ab der Mitte der 870er Jahre erbaut werden, als Arnolf „von Kärnten“ der tatsächliche Herr von Mosaburg war.

Mosaburg betrachtet man also zur Zeit Arnolfs auch seiner Rechtsstellung nach als eine $p f a l z,{ }^{36}$ also königliche Residenz. Es gehört in die Reihe jener neuen Königspfalzen (z. B. Paderborn, Trebur, Salz, Forchheim), die sich im 9. Jahrhundert neben den mehrmals erwähnten Hauptsitzen (Frankfurt/Main, Worms, Ingelheim und Regensburg) herausgebildet hatten. Auch in ihrem Fall kann man sich nicht allein auf die Schriftquellen verlassen, denn sie benutzen gleichwertig mit palatium die Begriffe villa, curtis, castrum, oppidum, civitas und urbs. ${ }^{37}$

Die historischen Quellen geben keine eindeutige Beschreibung über die Pfalz. Aufgrund der Biographien Einhards, Notkers und Wipos erweisen sich als grundlegende Bestandteile einer Pfalz das repräsentative Gebäude, die Kapelle, der häufige Besuch des Königs, die Abhaltung der Reichsversammlung und der Synode sowie die Abhaltung der kirchlichen und profanen Feiertage. Die Historiker benutzen den Ausdruck Pfalz allgemein in einem engeren Sinne und identifizieren sie mit der Königspfalz. Als solche definieren sie die Pfalz in spätantiker Bedeutung, d. h. sie verstehen darunter den Sitz und Ort der Macht des Herrschers, Wohnsitz und Rechtsort. In ihrer baulichen Erscheinung fasst die Königspfalz das Wohngebäude des Königs, die Pfalzkapelle, den Saalbau (aula) und den Wirtschaftshof in sich, also einen Wohn- und Rechtsbereich, einen Sakral- und einen Wirtschaftsbereich. ${ }^{38}$

Werner Jacobsen erkennt den entscheidenden Unterschied zwischen Pfalz (palatium) und Burg (castrum) darin, dass die Letztere unbedingt befestigt war, während die Befestigung bei Ersterer zwar ein wichtiges, aber nicht unbedingt erforderliches Kriterium war. Bei der Pfalz war eher jene Bereitschaft und Fähigkeit von Bedeutung, dass die Siedlung über repräsentativen, kunstfertig ausgeführten Wohn- und kirchlichen Gebäuden verfügte, die den Ansprüchen der Verwaltung, der Rechtsprechung und des Königs entsprachen. Man legt sicherlich auch Verteidigungseinrichtungen von gewissem Maße um sie an, sie sind jedoch wegen der mit der repräsentativen Funktion einhergehenden großen Flächenbeanspruchung nicht optimal $\mathrm{zu}$ verteidigen; sie können deshalb nur in friedlichen Zeiten ungestört fungieren. ${ }^{39}$

In den schriftlichen Quellen erscheint neben Mosaburg/ Zalavár auch Savaria/Szombathely als civitas, jedoch ist die karolingerzeitliche Siedlungsschicht Letzterer in solchem Maße zerstört und vernichtet, dass wir über Ausdehnung,

\footnotetext{
${ }^{36}$ Sennhauser (2011) 288-289.

${ }^{37}$ Die Quellenangaben siehe bei Sennhauser (2011) 286.

${ }^{38}$ Zur Zeit Ludwigs des Frommen umschrieb Wandalbert von Prüm die Pfalz als „Einrichtungen für den Aufenthalt des Königs und Ausrüstung für seine Reise“; vgl. Sennhauser (2011) 285.

${ }^{39}$ Jacobsen (1999) 109. - Vielsagend ist, dass die neu errichteten Pfalzen zur Zeit ihrer Erbauung noch als locus, villa erschienen und erst später taucht die Benennung palatium auf; vgl. Störmer (1995) 164-171.
} 
Charakter, Befestigung sowie inneren Aufbau der civitas keine Angaben haben. Wegen des Fehlens der geeigneten Fundstücke und anderer Siedlungsbefunde (Kirche, Palast, Wirtschaftsgebäude usw.) ist die Datierung der Burgmauer von Szombathely in die Karolingerzeit nur unter großem Vorbehalt anzunehmen. ${ }^{40}$

Das Arnolfinum ${ }^{41}$ erwähnt weitere Siedlungen in der östlichen Region als civitas: ${ }^{42}$ Magalicha/Melk (im Jahr 831 und 892 locus, 860 curtis), Pettouia/Pettau/Ptuj, Carantanum/Karnburg/Kranj, Treisma/Traismauer, Holunpurch/ Hollenburg a. d. Donau (860 curtis). ${ }^{43}$ Beachtenswert ist, dass nach der Urkunde ein Drittel mehrerer civitas $^{44}$ sich unmittelbar im Besitz des Erzbistums befindet oder dorthin gelangt. In der ursprünglichen Urkunde von 860 wird noch kein Wort über den civitas-Status dieser Siedlungen oder ihre innere Gliederung erwähnt. ${ }^{45}$ Da aber die Urkunde von 977 gefälscht und zurückdatiert wurde (?), ${ }^{46}$ gehören diese Folgen sicherlich in das 10. Jahrhundert. Die Gliederung der civitas in drei Teilen und der unterschiedliche Rechtsstatus der Einheiten könnten eine siedlungsgeschichtliche Phase der Entwicklung zu einer Stadt (Bischofs-, Erzbischofspfalzen?) anzeigen, welchen Abschnitt - nach der auch archäologisch dokumentierten Dreiteilung und Befestigungsarten der einzelnen Teile - Mosaburg/Zalavár bereits in der Karolingerzeit passierte und sich am Ende des 9. Jahrhunderts von einer Bischofspfalz zur Königspfalz erhob.

Die Siedlungsstruktur von Mosaburg ändert sich also verhältnismäßig schnell und sie weiht vom altmährischen Schema $a b$, als sie sich in Richtung einer für das Karolingische Reich charakteristischen frühen Stadt entwickelt. Demgegenüber haben die Zentren der altmährischen gentilen Fürsten einen eigenartigen, örtlichen Charakter. Sie entwickeln sich eher „horizontal“. In Mikulčice konzentrieren sich beispielsweise viel mehr Kirchen als in Mosaburg, da alle, mit Palisadenmauern umgrenzten, dicht nebeneinander gebauten Adelshöfe eine Eigenkirche haben, wo die Mitglieder der Stifterfamilie und ihre Dienstleute bestattet sind, sogar auch noch in der dritten Kirche, der sog. Basilika, die eventuell die Eigenkirche der Fürstenfamilie war. Wegen dieser Siedlungsstruktur konnten die altmährischen Zentren nicht weiter entwickeln und einen

\footnotetext{
${ }^{40}$ Tóth (1978); Kiss et al. (1998).

${ }^{41}$ MGH Diplom. Arnolfi 281-286, Nr. 184; MMFH III, 124-126, Nr. 110.

${ }^{42}$ Nach Dopsch (1989) 9-10 könnte bei der Bezeichnung mit civitas gelegentlich auch ein Hinweis auf eine römische Vergangenheit eine Rolle spielen.

${ }^{43}$ Reutter (1912) 16.

${ }^{44}$ MGH Diplom. Arnolfi 281-286: ad Magilicham terciam partem civitatis... quas quondam ad sanctum Emmerammum antecessores nostri tradiderunt [...] ad Holunpurch terciam partem civitatis [...] ad Pettouiam aecclesiam cum decima et duas partes civitatis ... et ex parte nostra addimus terciam partem civitatis, que proprietas Carantani fuit

${ }^{45}$ MGH Diplom. Ludwig d. D. 147-148, Nr. 102; MMFH III, 59-60.

${ }^{46}$ MGH Diplom. Arnolfi 281-282, Nr. 184 hält sie für authentisch; Koller (1969) argumentiert für die Fälschung der Urkunde; Dopsch (1989) 20 hält eine in mehreren Etappen erfolgte Fälschung für vorstellbar.
}

frühstädtischen Charakter vom westeuropäischen Sinne entfalten.

Nicht nur in der Titulatur des Hochadels, der Siedlungsstruktur der Zentren und der Aufgabe der Kirchen sind bedeutende charakteristische Unterschiede nachzuweisen, sondern auch in der Tracht und Bestattungsart in den Friedhöfen in und an der Kirche.

\section{DIE BESTATTUNGEN AN DER KIRCHE}

Im Gebiet der Grafschaft von Mosaburg galten die synodalen Verordnungen für die Bestattungen im Friedhof an der Kirche ebenso wie für jene im Reichsinneren. So wie die Reichsaristokratie und vom Beginn des 9. Jahrhunderts an bereits auch die sächsische Elite keine wertvollen Schmucksachen, rangabzeichnenden Trachtbestandteile oder Sporen mehr bestatten, wird ihr Rang höchstens von einem sorgfältig gezimmerten, mit Eisenbändern zusammengehaltenen, großen Sarg angedeutet. So ist auch für die Mehrheit der Gräber der die christlichen Vorschriften eifrig einhaltenden, vermögenderen Mosaburger das vollkommene Fehlen der Beigaben charakteristisch. Auf ihren Rang deutet allein der privilegierte Grabplatz neben der Kirche, die Art des Grabbaus und die Größe und Ausführung des Sarges hin.

Den Friedhof um Hadrians Wallfahrtskirche gliederte man mit Steinmauern und Holzpalisaden in kleinere Familieneinheiten. Die mit Steinmauer abgegrenzten Familiengrüfte sind nicht nur unter den pannonischen Kirchen, sondern auch im ganzen Karolingerreich einzigartig. Diese nehmen nicht nur die Räume zwischen den aus der Umgangskrypta sich öffnenden drei kleinen Altarräumen ein, sondern verlaufen entlang der nördlichen und südlichen Seite der Kirche. Dass hier die Vornehmsten ruhen, belegen die gemauerten Gräber, die man teils aus Quaderstein und teils aus amorphem Sandstein, Kalkstein und Basalt sowie aus Ziegelstein mit Verwendung von Kalkmörtel sorgfältig errichtete. In einigen Fällen wurden sie im Inneren auch noch verputzt (?) und mit Mörtelboden beschichtet, die innerhalb oder in unmittelbarer Nähe dieser Räume vorkamen. ${ }^{47}$

Für die nur in Ausnahmefällen mit reichen Beigaben bestatteten Vornehmen zimmerte man den Sarg aus dicken Brettern und hielt ihn mit Eisennägeln und -klammern zusammen, ${ }^{48}$ es wurden aber keine für die Bestattungen der altmährischen Aristokratie so charakteristischen Eisenbeschläge benutzt. ${ }^{49}$ Man hob jedoch als Erleichterung für das Herunterlassen der Särge quer an den beiden Enden der Grabgrube in der Awarenzeit oft verwendete schmale Graben aus. Die Gräberfelder der Kirchen foris civitatem (Récéskút und Zalaszabar-Borjúállás-Insel) werden von weniger anspruchsvoll gebauten Gräbern mit

\footnotetext{
${ }^{47}$ Sanke (2010) 135.

${ }^{48}$ Klima (1983).

${ }^{49}$ Poláček (2005); Galuška (2005).
} 
wenigen Beigaben charakterisiert. In einer Hinsicht sind aber alle Kirchen ähnlich: Weder auf der Burginsel noch in den Kirchen außerhalb dieser wurde innerhalb der Kirche bestattet. $^{50}$ Die Gräber des Bauherres/Stifters und dessen Familienmitglieder wurden außerhalb der Kirche angelegt und sie stechen allein dadurch hervor, dass sie ausgesprochen separat liegen, wie z. B. zwischen den Altarräumen der Hadrianskirche mit einer Steinmauer abgegrenzt oder auf der Zalaszabar-Borjúállás-Insel im Narthex vor dem westlichen Eingang des Kirchenschiffes.

Die Männergräber der Oberschicht bei den Kirchen von Mosaburg beinhalten ein von den endawarenzeitlichen Bestattungen des militärischen Gefolges radikal abweichendes Fundmaterial. Waffen legte man fast keinem der um die Mosaburger Kirchen angelegten Männergräber bei. ${ }^{51}$ Auch von den „Scramasaxen“52 in zwei Gräbern (Grab 292 und 322) bei der Marienkirche stellte sich heraus, dass sie große Jagdmesser auf den beiden Seiten der Klinge mit Blutrinne in einer Messerscheide sog. baltischer Konstruktion sind. Ein ähnliches Eisenmesser fand sich auch im Gräberfeld neben der Hadrianskirche; ${ }^{53}$ sie kommen aber verhältnismäßig oft sogar in den Gräberfeldern des Gemeinvolkes vor. ${ }^{54}$ Es gibt eine einzige Ausnahme. Im Grab 20/19 bei der Marienkirche lag ein junger Mann, der wahrscheinlich dem engeren Familienkreis von Priwina und Chezil angehörte. Er wurde mit einem Schwert mit silbertauschiertem und silberplattiertem, bronzegegossenem Gefäß und mit einer Sporengarnitur bestattet. ${ }^{55}$

Die Bestattungen des Adels von Mosaburg scheinen sich von denen der Reichsaristokratie lediglich darin zu unterscheiden, dass der Mosaburger Adel das christliche Prinzip der Fürsorge für die Toten, wonach man das Opfer zur Rettung der Seele der Kirche und den Armen anstelle der Grabbeigabe darbietet, ${ }^{56}$ bei jungen, früh verstorbenen Mitgliedern gewisser Familien ziemlich konsequent nicht einhält. Anstelle der Verpflichtung gegenüber der Kirche oder darüber hinaus? - bestatteten sie ihre jungen Toten mit Trachtzubehör. Die weiblichen Toten waren mit einer

\footnotetext{
${ }^{50}$ Über die Bestattungen innerhalb der Kirchen in Mähren und Böhmen aus dem 9.-10. Jahrhundert siehe Schulze-Dörrlamm (1993); Kavánová (2005); Poláček (2005, 140).

${ }^{51}$ In der Nähe des Grabes 265 im Abschnitt XXII bei der Marienkirche in Zalavár-Burginsel fand sich eine einfache Lanzenspitze, die zu keinem Grab gehörte, sondern als Streufund aufgelesen wurde; vgl. Sós and Bökönyi (1963) 67, Taf. XCI.1-2.

${ }^{52}$ Sós and Bökönyi (1963) 45, 47.

${ }^{53} \mathrm{Im}$ Grab 410/91 fand sich ein großes Jagdmesser in einer Messerscheide baltischer Konstruktion mit eisernen Ortbandbeschlägen, die mittels Nieten, die mit Perldrahtkranz umgeben wurden, verziert und befestigt wurden.

${ }^{54}$ Szőke et al. (1992) 81-83.

${ }^{55}$ Die Restaurierung des Schwertes ist noch nicht abgeschlossen, es handelt sich aber um ein Schwert, dessen beste Analogien sich in Skandinavien und besonders in Haithabu befinden; vgl. Arents and Eisenschmidt (2010) 75-76.

${ }^{56}$ Angenendt (2008) 316.
}

Haube mit silbervergoldetem Kopfschmuck und Perlenkette, goldenen und silbernen Fingerringen und Blechknöpfen verziert. ${ }^{57}$ Die Männer wurden mit Beutel (Ahle, Feuergerät) und als Essbesteck benutztem Eisenmesser (oft paarweise in den Gräbern), ferner an den Füßen mit Sporengarnitur oder - seltener - mit Wadenbinde- oder Schuhgarnitur bestattet.

In den Friedhöfen an der Marien- und der Hadrianskirche sind Sporengarnituren lediglich in etwa einem Dutzend Gräbern freigelegt worden. Interessant ist, dass ähnlich den Frauengräbern auch bei den Männern die Sporen nur in den juvenilen, frühadulten Gräbern beigesetzt wurden - während im Fall der Bestattungen der Dienstleute auf dem Einzugsgebiet Mosaburgs der Sporn zur Tracht jener Erwachsenen gehörte, die in der Gemeinschaft eine entscheidende Führungsrolle spielten.

Dagegen waren die Männer der altmährischen führenden Schicht viel häufiger mit Sporengarnitur, nicht einmal auch mit Schwert und mit Bartaxt oder Lanze bestattet. Diese Gräber kamen an den vornehmsten Stellen des Friedhofes oder sogar innerhalb der Kirche vor. Diese Tatsache bestätigt die Vermutung, dass diese Männer nur zum gentilen Adel des altmährischen Fürstentums gehören konnten, der die christlichen Vorschriften und synodalen Verordnungen viel weniger eifrig einhielt, als die Elite von Mosaburg.

Zusammenfassend kann man feststellen, dass sich im östlichen Randgebiet des Karolingischen Reiches zwei verschiedene Gruppen der Elite herausbildeten. Was ihre Namensverwendung betrifft, wird die eine Gruppe nur mit Personennamen oder mit dem Namen des von ihnen verwalteten Gebietes erwähnt, die andere mit dem Namen ihres Volkes. Die Mitglieder der ersten Gruppe verwalteten die Einheiten des Kerngebietes des ehemaligen awarischen Khaganats als die karolingischen Hauptamtsträger und königlichen Vasallen im Reichsinneren. Die der zweiten waren die mittelbaren Verbündeten der Awaren und hatten einen eigenen Stammesfürsten und gentile Adeligen. Die Entwicklung der Verwaltungszentren der unmittelbar zum Karolingischen Reich gehörenden Provinz Pannoniae läuft mehr oder weniger synchron mit der der Zentren im Reichsinneren, während die Herrensitze außerhalb der Provinz eine spezifische Siedlungsstruktur mit Adelshöfen der militärischen Gefolgschaft des Stammesfürsten aufweisen. Dementsprechend sind die Kirchen innerhalb dieser immer Eigenkirchen, wo die Stifterfamilie mit den $\mathrm{zu}$ ihr gehörenden Personen bestattet wird (nicht einmal auch innerhalb der Kirche). Sie hielten die christlichen Vorschriften eben deshalb viel weniger ein - siehe auch die Gräber mit Waffenbeigaben an oder in der Kirche -, als in den östlichsten Grafschaften des Karolingischen Reiches. Die drei Kirchen in Mosaburg hatten unterschiedliche Aufgaben und ihre Einheit bildet eine spezielle Dreikirchengruppe, eine bischöfliche Kirchenfamilie.

${ }^{57}$ Wie z. B. in den Gräbern 157/99, 159/99 an der Nordseite der Kirche. 


\section{REFERENCES}

Angenendt, A. (2008). Das Frühmittelalter. Die abendländische Christenheit von 400 bis 900. Stuttgart-Berlin-Köln.

Arents, U. and Eisenschmidt, S. (2010). Die Gräber von Haithabu. Die Ausgrabungen in Haithabu 15/1-2. Neumünster.

Bóna, I. (1984). A népvándorláskor és a korai középkor története Magyarországon [Die Geschichte der Völkerwanderungszeit und des frühen Mittelalters in Ungarn]. In: Bartha, A. (Hrsg.), Magyarország története, I.: Elözmények és magyar történet 1242-ig. Budapest, pp. 265-373, 1586-1606.

Czeglédy, K. (1967). Megjegyzések a honfoglalás előtti magyar királyság intézményéhez [Bemerkungen zur Institution des ungarischen Königtums vor der Landnahme]. In: Imre, S., and Szathmári, I. (Hrsg.), A magyar nyelv története és rendszere. Nyelvtudományi értekezések 58. Budapest, pp. 86-87.

Dopsch, H. (1989). Von der Slawenmission zur Grundherrschaft. Zur Rolle des Erzbistums Salzburg und der Salzburger Klöster in Niederösterreich. In: Feigl, H., et al. (Hrsg.), Die bayerischen Hochstifte und Klöster in der Geschichte Niederösterreichs. Vorträge und Diskussionen des siebenten Symposions des Niederösterreichischen Instituts für Landeskunde, Waidhofen an der Ybbs, 7.-9. Juli 1986. Studien und Forschungen aus dem Niederösterreichischen Institut für Landeskunde 11. Wien, pp. 1-30.

Galuška, L. (2005). Gehörten die in Särgen bestatteten Personen zur Gesellschaftselite des großmährischen Staré Město - Uherské Hradiště? In: Kouřil (2005), pp. 193-208.

Györffy, Gy. (Hrsg.) (1958). A magyarok elödeiröl és a honfoglalásról. Kortársak és krónikások híradásai [Über die Vorfahren der Ungarn und die Landnahme. Benachrichtigung von Zeitgenossen und Chronisten]. Nemzeti könyvtár - történelem. Budapest.

Hellmann, M. (1964). Der Begriff „populus“ in der Conversio Bagoariorum et Carantanorum. In: Hellmann, M., Olesch, R., Stasiewski, B., and Zagiba, F. (Hrsg.), Cyrillo-Methodiana. Zur Geschichte des Christentums bei den Slawen 863-1963. Slawistische Forschungen 6. Köln-Graz, pp. 161-167.

Jacobsen, W. (1999). Pfalzen. In: von der Deutschen Burgenvereinigung e. V. durch Böhme, H.W., von Dollen, B., Kerber, D., Meckseper, C., Schock-Werner, B., and Zeune, J. (Hgg.), Burgen in Mitteleuropa. Ein Handbuch, I.: Bauformen und Entwicklung. Stuttgart, pp. 109-127.

Kahl, H.-D. (2003). Die Karolingerpfalz Karnburg. In: Hesse, Ch., Immenhauser, B., Landolt, O., and Studer, B. (Hrsg.), Personen der Geschichte - Geschichte der Personen. Studien zur Kreuzzugs-, Sozial- und Bildungsgeschichte. Festschrift für Rainer Christoph Schwinges zum 60. Geburtstag. Basel, pp. 365-392.

Kavánová, B. (2005). Großmährische Gräber in Kirchen Erkenntnisstand. In: Kouřil (2005), pp. 49-65.

Kiss, G., and Tóth, E. (1993). A szombathelyi Szent Mártontemplom régészeti kutatása 1984-1992. Előzetes jelentés a feltárt 9-13. századi emlékekről (Archäologische Untersuchung der St. Martinskirche in Szombathely 1984-1992. Vorläufiger Bericht der freigelegten Denkmäler aus dem 9.-13. Jahrhundert). ComArchHung, 1993: 175-199.

Kiss, G., Tóth, E., and Zágorhidi Czigány, B. (1998). SavariaSzombathely története a város alapitásától 1526-ig [Die
Geschichte von Savaria/Szombathely von der Stadtgründung bis 1526]. Szombathely.

Klima, B. (1983). Železné skoby z Mikulčic (Eisenklammern [Eisenkrampen] aus Mikulčice). AR, 35: 172-188.

Koller, H (1969). König Arnulfs großes Privileg für Salzburg. MGSL, 109: 65-75.

Koller, F (1988). Die Anfänge der Salzburger Städte. Civitas und verwandte Begriffe in den Salzburger Quellen. MGSL, 128: 5-31.

Kouřil, P. (Hrsg.) (2005). Die frühmittelalterliche Elite bei den Volkern des östlichen Mitteleuropas (mit einem speziellen Blick auf die großmährische Problematik). Materialien der internationalen Fachkonferenz Mikulčice 25.-26. 5. 2004. Brno.

Lehmann, E. (1952/53). Die entwicklungsgeschichtliche Stellung der karolingischen Klosterkirche zwischen Kirchenfamilie und Kathedrale. Wissenschaftliche Zeitschrift der Friedrich-SchillerUniversität Jena, 1952/53: 131-144.

Lehmann, E. (1962). Von der Kirchenfamilie zur Kathedrale. Bemerkungen $\mathrm{zu}$ einer Entwicklungslinie der mittelalterlichen Baukunst. In: Schmoll gen. Eisenwerth, J.A. (Hrsg.), Varia Formae Veritas Una. Kunsthistorische Studien. Festschrift Friedrich Gerke. Baden-Baden, pp. 21-37.

Lošek, F. (1997). Die Conversio Bagoariorum et Carantanorum und der Brief des Erzbischofs Theotmar von Salzburg. MGH Studien und Texte 15. Hannover.

MMFH Havlík, L. (Ed.) (1966-1977). Magnae Moraviae Fontes Historici, I-V. Praha-Brno.

Pohl, W. (1988). Die Awaren. Ein Steppenvolk in Mitteleuropa 567822 n Chr. München.

Poláček, L. (2005). Zur Erkenntnis der höchsten Eliten des großmährischen Mikulčice. Gräber mit beschlagenen Särgen. In: Kouřil (2005), pp. 137-156.

Poláček, L. (2009). Die Kirchen von Mikulčice als Spiegel von Glaube und Herrschaft. In: von Freeden, U., Friesinger, H., and Wamers, E. (Hrsg.), Glaube, Kult und Herrschaft. Phänomene des Religiösen im 1. Jahrtausend n. Chr. in Mittel- und Nordeuropa. Akten des 59. Internationalen Sachsensymposions und der Grundprobleme der frühgeschichtlichen Entwicklung im Mitteldonauraum. Römisch-Germanische Kommission, Frankfurt a. M. - Eurasien-Abteilung des Deutschen Archäologischen Instituts, Berlin. Kolloquien zur Vor- und Frühgeschichte 12. Bonn, pp. 417-435.

Rau I. Rau, R. (1968-1987). Quellen zur karolingischen Reichsgeschichte, I.: Die Reichsannalen, Einhards Leben Karls des Großen, zwei „Leben“ Ludwigs, Nithardc Geschichten. Ausgewählte Quellen zur deutschen Geschichte des Mittelalters 5. Darmstadt.

Rau III. Rau, R. (1969-1982). Quellen zur karolingischen Reichsgeschichte, III.: Jahrbücher von Fulda, Reginos Chronik, Notkers Taten Karls. Ausgewählte Quellen zur deutschen Geschichte des Mittelalters 8. Darmstadt.

Reutter, H. (1912). Ein fränkisches Grenz- und Siedlungssystem in den karolingischen Südostmarken. JLNÖ, 10(1911): 1-108.

Sanke, M. (2012). Die Gräber geistlicher Eliten Europas von der Spätantike bis zur Neuzeit. Archäologische Studien zur materiellen Reflexion von Jenseitsvorstellungen und ihrem Wandel. ZfAM Beiheft 25. Bonn.

Schulze-Dörrlamm, M (1993). Bestattungen in den Kirchen Großmährens und Böhmens während des 9. und 10. Jahrhunderts. JRGZM, 40: 557-620. 
Sennhauser, H.R. (2011). Vom palatium zur Bischofspfalz. Zusammenschau. In: Sennhauser, H.R. (Hrsg.): Pfalz - Kloster Klosterpfalz St. Johann in Müstair. Historische und archäologische Fragen. Tagung 20.-22. September 2009 in Müstair. Acta Müstair, Kloster St. Johann 2. Zürich.

Sós, Á., and Bökönyi, S. (1963). Die Ausgrabungen Géza Fehérs in Zalavár. ArchHung 41. Budapest.

Cs. Sós, Á. (1973). Die slawische Bevölkerung Westungarns im 9. Jahrhundert. Münchner Beiträge 22. München.

Štih, P. (1994). Priwina: Slawischer Fürst oder fränkischer Graf? Ethnogenese und Überlieferung. VIÖG, 31: 209-222.

Štih, P. (2010). The Middle Ages between the Eastern Alps and the Northern Adriatic. Selected papers on Slovene historiography and medieval history. East Central and Eastern Europe in the Middle Ages, 450-1450, 11. Leiden-Boston.

Störmer, W. (1995). Karolingische Pfalzen in Franken. In: Kolmer, L., and Segl, P. (Hrsg.), Regensburg, Bayern und Europa. Festschrift für Kurt Reindel zu seinem 70. Geburtstag. Regensburg, pp. 161-173.

Szőke, B.M., Éry, K., Müller, R., and Vándor, L. (1992). Die Karolingerzeit im unteren Zalatal. Gräberfelder und Siedlungsreste von Garabonc I-II und Zalaszabar-Dezsősziget. Antaeus 21. Budapest.
Szücs, J. (1997). A magyar nemzeti tudat kialakulása [Die Herausbildung des ungarischen Nationalbewusstseins]. Budapest.

Tellenbach, G. (1979). Die geistigen und politischen Grundlagen der karolingischen Thronfolge. Zugleich eine Studie über kollektive Willensbildung und kollektives Handeln im neunten Jahrhundert. FMSt, 13: 184-302.

Tóth, E. (1978). Die karolingische Burg von Sabaria-Szombathely. FolArch, 29: 151-182.

Wolfram, H. (1979). Conversio Bagoariorum et Carantanorum. Das Weißbuch der Salzburger Kirche über die erfolgreiche Mission in Karantanien und Pannonien, Wien-Köln-Graz.

Wolfram, H. (1986). Slawische Herrschaftsbildungen im pannonischen Raum als Voraussetzung für die Slawenmission. In: Dopsch, H. (Hrsg.), Salzburg und die Slawenmission. Zum 1100. Todestag des hl. Methodius. Beiträge des internationalen Symposions vom 20. bis 22. September 1985 in Salzburg. MGSL, 126: 245-253.

Wolfram, H. (1995). Grenzen und Räume. Geschichte Österreichs vor seiner Entstehung. Österreichische Geschichte 378-907. Wien.

Wolfram, H. (1996). Salzburg, Bayern, Österreich. Die Conversio Bagoariorum et Carantanorum und die Quellen ihrer Zeit. MIÖG Ergänzungsband 31. Wien-München. 